\title{
The Theory of Stochastic Space-Time. 1. Gravitation as a Quantum Diffusion ${ }^{1}$
}

\begin{abstract}
Zahid Zakir $^{2}$
Abstract

The Nelson stochastic mechanics of inhomogeneous quantum diffusion in flat spacetime with a tensor of diffusion can be described as a homogeneous one in a Riemannian manifold where this tensor of diffusion plays the role of a metric tensor multiplied to the diffusion coefficient. It is shown that such diffusion accelerates both a sample particle and local inertial frames such that their mean accelerations do not depend on their mass. This fact, explaining the principle of equivalence, allows one to represent the curvature and gravitation as consequences of the inhomogeneity of the quantum fluctuations. In this diffusional treatment of gravitation it can be naturally explained the fact that the energy density of the instantaneous Newtonian interaction is negative defined (with respect to a point at spatial infinity).
\end{abstract}

PACS: 04.20.Cv, 03.65.Ta, 05.40.Jc, 04.62.+v

Key words: stochastic mechanics, tensor of diffusion, quantum fluctuations, gravitation, curvature

\section{Content}

Introduction 1

1. Stochastic mechanics of homogeneous diffusion .......................................................... 2

2. Stochastic mechanics of inhomogeneous diffusion ................................................. 3

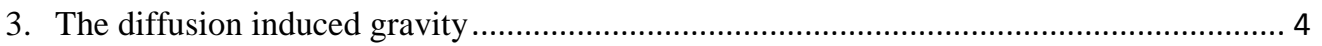

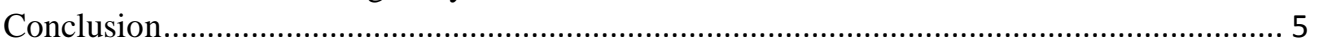

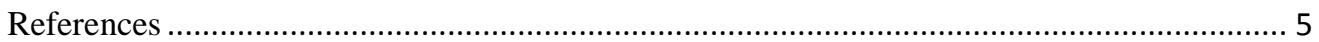

\section{Introduction}

Two basic phenomena of physics - gravitation and quantum fluctuations - both depend on masses of objects only and both have a geometric nature. A geometrical origin of gravitation is well known, while up to now Nelson's discovery of the stochastic geometry of spacetime [1] does not accepted as one of fundamental concepts of physics. The stochastic mechanics is unique version of quantum mechanics where the quantum fluctuations are represented as a manifestation of stochastic geometry of spacetime with a constant diffusion coefficient $v_{0}=\hbar / 2 m$ (see also [2-5]).

It is natural to consider a more general case of spacetimes with inhomogeneous diffusion by a tensor of diffusion $v^{a b}(x, t)$. In the paper it will be shown that the stochastic structure of the spacetime with such tensor of diffusion induces a nontrivial metric $g^{a b}(x, t)$ and a curvature. This means that gravitation can be treated as a quantum diffusional effect.

\footnotetext{
${ }^{1}$ The preprint of the paper has been presented in 1998: Zakir Z. arXiv:hep-th/9812254.

${ }^{2}$ Centre for Theoretical Physics and Astrophyics, Tashkent,Uzbekistan; zahidzakir@theor-phys.org
} 
The fact that the quantum fluctuations and gravitation are not independent phenomena (see also [5]), leads to the interesting solution of the problem of the gravitational energy. It is well known that the energy density of the instantaneous Newtonian interaction, as only attractive one, is negative defined. In the diffusional treatment of gravity this property can be naturally explained.

\section{Stochastic mechanics of homogeneous diffusion}

Let we have Nelson's diffusion [1] of a non-relativistic particle of mass $m$ in Euclidean space $R^{n}$ :

$$
d x_{ \pm}^{a}(t)=b_{ \pm}^{a}(x, t) d t+d w_{ \pm}^{a}(t)
$$

with

$$
\begin{gathered}
\lim _{\Delta t \rightarrow 0} E\left[\Delta w_{ \pm}^{a}(t) \mid x^{a}(t)\right]=0 \\
\lim _{\Delta t \rightarrow 0} E\left[\Delta w_{ \pm}^{a}(t) \Delta w_{ \pm}^{b}(t) \mid x^{a}(t)\right]= \pm 2 v_{0} \delta^{a b} d t .
\end{gathered}
$$

Here the diffusion coefficient is taken as $v_{0}=\hbar / 2 m$, where $\hbar$ is the Planck constant, $\Delta w_{ \pm}^{a}(t)=w^{a}(t \pm \Delta t)-w^{a}(t)$ and the limit $\Delta t \rightarrow 0$ should be taken only after the calculation of the conditional expectations $E\left[\ldots \mid x^{a}(t)\right]$. The drifts:

$$
b_{ \pm}^{a}(x, t)=\lim _{\Delta t \rightarrow 0} E\left[\frac{\Delta x_{ \pm}^{a}(t)}{ \pm \Delta t} \mid x^{a}(t)\right]
$$

allow one to define the current $\left(v^{a}\right)$ and osmotic $\left(u^{a}\right)$ velocities:

$$
v^{a}=\frac{1}{2}\left(b_{+}^{a}+b_{-}^{a}\right), \quad u^{a}=\frac{1}{2}\left(b_{+}^{a}-b_{-}^{a}\right) .
$$

The acceleration is defined as:

$$
\begin{aligned}
a^{a}(x, t) & =\frac{1}{2}\left(D_{+} D_{-}+D_{-} D_{+}\right) x^{a}(t)= \\
& =\frac{\partial v^{a}}{\partial t}+(\mathbf{v} \nabla) v^{a}-(\mathbf{u} \nabla) u^{a}-v_{0} \Delta u^{a},
\end{aligned}
$$

where the stochastic derivatives $D_{ \pm}$are:

$$
\begin{aligned}
D_{ \pm} f & =\lim _{\Delta t \rightarrow 0} E\left[\frac{f[x(t \pm \Delta t), t \pm \Delta t]-f(x, t)}{ \pm \Delta t} \mid x^{a}(t)\right] \\
& =\frac{\partial f}{\partial t}+\left(\mathbf{b}_{ \pm} \nabla\right) f \pm v_{0} \Delta f .
\end{aligned}
$$

The mean trajectories of free classical particles by the current velocity $\mathbf{v}$ are diffusional geodesic lines on $R^{n}$. The equation of motion for them in the external field is the Newton equation:

$$
m \mathbf{a}=-\nabla V .
$$

The mean value of the acceleration is:

$$
E\left[a^{b}(t)\right]=\int d x \rho(x, t) a^{b}(x, t)=\int d x \rho\left[\frac{\partial v^{b}}{\partial t}+(\mathbf{v} \nabla) v^{b}\right] \rho,
$$

which for the Newtonian potential $\phi_{N}$ gives: 


$$
E\left[\frac{\partial v^{b}}{\partial t}+(\mathbf{v} \nabla) v^{b}\right]=-\nabla^{b} \phi_{N} .
$$

Here the mean acceleration of the sample particle in the static gravitational field $\phi_{N}$ does not depend on mass of this particle in accordance with the principle of equivalence.

\section{Stochastic mechanics of inhomogeneous diffusion}

Let us consider in $R^{n}$ a general diffusion with the tensor of diffusion:

$$
v_{a b}(x, t)=v_{0} \gamma_{a b}(x, t),
$$

where $\gamma_{a b}$ is a normalized tensor of diffusion which in the case of Nelson's homogeneous diffusion has been taken as: $\gamma_{a b}=\delta_{a b}$. The mean trajectories of particles in this general case are not geodesic lines on $R^{n}$, but contain some deviations from the geodesics. For the description of the general diffusion we introduce the curvilinear coordinates $x^{i}\left(x^{a}, t\right)$ and basis vectors $e_{i}^{a}$ along the mean trajectories of the free particles. Then we have for the local physical coordinates of the sample particle at a point $M$ :

$$
\begin{gathered}
d x^{i}(M, t)=e_{a}^{i}(M, t) d x^{a}(M, t), \\
d x_{ \pm}^{a}(t)=b_{ \pm}^{a}(x, t) d t+d w_{ \pm}^{a}(t),
\end{gathered}
$$

where $e_{i}^{a} e_{i}^{b}=\delta^{a b}, e_{i}^{a} e_{j}^{a}=g_{i j}$, and $g^{a b}=e_{i}^{a} e_{j}^{b} g^{i j}$ is the metric of flat spacetime $R^{n}$, $g^{i j}(x, t)$ is the metric tensor of the manifold $M$ with the curvilinear coordinates $x^{i}$, formed by the mean trajectories of the current velocity $v^{i}(x, t)$ at the free diffusion:

$$
d x_{ \pm}^{i}(t)=b_{ \pm}^{i}(x, t) d t+d\left[e_{a}^{i}(x, t) w_{ \pm}^{a}(t)\right] .
$$

Then the conditional expectations can be defined in terms of these curvilinear coordinates only for small temporal intervals $\Delta t$ and along a piece-wise smooth curve approximating random curves near the point $M$. The corresponding tensor of diffusion is defined as:

$$
v^{i j}(x, t)=\frac{1}{2} \lim _{\Delta t \rightarrow 0} E_{\gamma}\left[\frac{\Delta x_{ \pm}^{i}(t) \Delta x_{ \pm}^{j}(t)}{ \pm \Delta t} \mid x^{i}(t)\right]=v_{0} \gamma^{i j}(x, t) .
$$

Let us introduce a new type of the parallel transport of tensors - the stochastic parallel transport in flat spacetime along the mean trajectory of the free drift:

$$
d e_{a}^{i}(x, t)=-\Gamma_{m l}^{i} e_{a}^{l} d x^{m}(t)-\frac{1}{2} d\left[\Gamma_{m l}^{i} e_{a}^{l}\right] d x^{m}(t) .
$$

Such inhomogeneous diffusion can be described as a homogeneous one in the Riemannian manifold with a constant diffusion coefficient $v_{0}=$ const and the metric tensor $g^{i j}(x, t)$ identified by the normalized tensor of diffusion: $\gamma^{i j}(x, t)=g^{i j}(x, t)$. Then stochastic mechanics with the tensor of diffusion can be treated as quantum mechanics in Riemannian manifold. Therefore, one can use the well-known formulas of the stochastic mechanics in curved manifolds [3].

The stochastic derivatives have the form:

$$
\left(D_{ \pm} F\right)^{i}(x, t)=\frac{\partial F^{i}}{\partial t}+\left(\mathbf{b}_{ \pm} \nabla\right) F^{i} \pm v_{0}\left(\Delta_{D R} F\right)^{i},
$$

where $\nabla$ is the Laplace-Beltrami operator in the curved manifold and: 


$$
\left(\Delta_{D R} F\right)^{i}=\Delta F^{i}+R_{j}^{i} F^{j} .
$$

is the Laplace-de Rham operator, $\Delta=\nabla \nabla$, and $R_{j}^{i}$ is the Ricci tensor.

From the expression for the acceleration

$$
a^{i}(x, t)=\frac{1}{2}\left(D_{+} b_{-}+D_{-} b_{+}\right)^{i}(x, t)=-\frac{1}{m} \nabla^{i} V
$$

and (8) one can obtain the equations of motion:

$$
\frac{\partial v^{i}}{\partial t}+(\mathbf{v} \nabla) v^{i}-(\mathbf{u} \nabla) u^{i}-v_{0} \Delta u^{i}-v_{0} R_{j}^{i} F^{j}=-\frac{1}{m} \nabla^{i} V
$$

The continuity equation for the probability density $\rho(x, t)$ :

$$
\frac{\partial \rho}{\partial t}+\nabla_{i}\left(\rho v^{i}\right)=0
$$

gives $u^{i}=v_{0}\left(\nabla^{i} \rho\right) / \rho$. Then by means of Nelson's assumptions [1] $m v^{i}=\nabla^{i} S$, $v_{0}=\hbar / 2 m$ and:

$$
\psi(x, t)=\sqrt{\rho(x, t)} \exp [i S(x, t) / \hbar],
$$

where $S(x, t)$ is some function and $\psi(x, t)$ is the wave function, we obtain the Schrödinger equation for particle's motion in the stochastic space with the tensor of diffusion $[1,3]$ :

$$
i \hbar \frac{\partial \psi}{\partial t}=-\frac{\hbar^{2}}{2 m} \Delta \psi+V \psi
$$

\section{The diffusion induced gravity}

The mean value of acceleration does not contain the terms with the osmotic velocity $u^{i}$ and although no any external field, nevertheless, there appears a proper acceleration due to the presence of the derivatives of the metrics in the Laplace-Beltrami operator:

which means that:

$$
E\left[\frac{\partial v_{i}}{\partial t}+(\mathbf{v} \nabla) v_{i}\right]=0
$$

$$
E\left[\frac{\partial v_{i}}{\partial t}+v^{j} \partial_{j} v_{i}\right]=E\left[\Gamma_{i j}^{k} v^{j} v_{k}\right]
$$

This a diffusion induced mean acceleration does not contain a dependence on mass of the particle, i.e. we have an analog of the equivalence principle as for gravitation.

The stochastic mechanics has been naturally generalized to the case of relativistic particle [4]. In this case we obtain non-trivial metrics for spacetime, which we can interpret as diffusion induced gravity. In other words, inhomogeneous diffusion leads to the acceleration of the particle exactly the same as some effective gravitational field.

It is very important that independence of the acceleration on the mass of the sample particle leads also to the same proper acceleration of macroscopic objects basises of local inertial frames. The acceleration of the reference frame means the appearance of non-trivial metrics and non-zero curvature. Einstein's equations for the curvature and the metric tensor then can be represented as equations for the tensor of diffusion. 
The identification of the metric structure of spacetime with the general diffusion leads to the interpretation of gravitation as a secondary effect of the quantum fluctuations.

In the stochastic interpretation of gravitation the equations for the metrics $g_{i j}(x, t)$ can be obtained from the diffusion equations. The gravitation is the energymomentum effect and we deal with some analog of a thermo-diffusion, where the diffusional flow $\mathbf{j}$ is directed opposite to the gradient of the vacuum energy density in space:

$$
\mathbf{j}=-\alpha \cdot \nabla \rho_{(0)},
$$

where $\alpha$ is some constant. Let a sample particle is at rest at very large distance from the source, where the energy density of the vacuum is $\rho_{(0) 1}$. Due to the inhomogeneous diffusion, the particle will move to the massive source, where the energy density is $\rho_{(0) 2}$. The diffusion leads to the drift of the sample particle from the intensive fluctuating region of the background to the lower intensity region. Therefore, for the attractive diffusion the difference of the energy densities $\delta \rho_{2-1}=\rho_{(0) 2}-\rho_{(0) 1}$ should be negative $\delta \rho_{2-1}<0$.

Therefore, if we interpret the gravitation as such diffusion process, the energy density corresponding to the gravitational interaction, associating by $\delta \rho_{2-1}$, must be negative defined. The fact that the energy density of the instantaneous Newtonian interaction is negative defined, is well known and we can treat this property of gravitation as one of evidences of its diffusional origin. Here matter's energy-momentum density tensor $T_{i j}$ plays the role of a source for the deformations of the stochastic structure of spacetime.

Thus, there is some dualism between the deformations of the stochastic structure and the Riemannian structure of spacetime.

\section{Conclusion}

Main result of the paper is the fact, that the quantum fluctuations induces gravitation and that the gravitation can be treated as a macroscopic remainder of the inhomogeneous quantum fluctuations. This result is very important not only for the understanding of the physical nature of gravitation, but for the strategy of construction of unified theories also. If, in the stochastic treatment, gravitation and quantum fluctuations are not independent phenomena, then it becomes clear that the unified theories must lead to an explanation of this fundamental fact.

In the next paper [6] it will be shown that the stochastic treatment of quantum theory and gravitation are not only some hypotheses, but can be considered as consequences of some general principles of invariance.

\section{References}

1. Nelson E. (1966) Phys.Rev., 150, p. 1079.

2. Blanchard Ph., Combe Ph., Zheng W. (1987) Mathematical and Physical Aspects of Stochastic

Mechanics, Lect. Not. in Phys., 281, 171 p.

3. Dohrn D., Guerra F. (1978) Lett. Nuovo Cim., 22, 4, p. 121.

4. Guerra F., Ruggiero P. (1978) Lett. Nuovo Cim., 23, 15, p. 529.

5. Smolin L. (1986) Phys.Lett., 113A, No 8, p. 408.

6. Zakir Z. (2009) Theor. Phys., Astrophys. and Cosmol., 4, 2, p. 11.

The Theory of Stochastic Space-Time. II. Quantum Theory of Relativity, arXiv:hep-th/9901013 